Instituto Internacional de Investigación y Desarrollo Tecnológico Educativo INDTEC, C.A.

DOI: https://doi.org/10.29394/scientific.issn.2542-2987.2017.0.0.10.174-193

OAI-PMH: http://www.indteca.com/ojs/index.php/Revista Scientific/oai

\title{
Preservación del Paisaje Cultural Arqueológico en la Comunidad de San Antonio Municipio Pedraza Año 2017
}

(Proyecto en ejecución)

\author{
Autora: Isabel Galeano Torres \\ Universidad Pedagógica Experimental Libertador, UPEL \\ 22marije@gmail.com \\ Barinas, Venezuela
}

\section{Resumen}

El presente estudio tendrá como propósito, desarrollar un plan acción para la Preservación del Paisaje Cultural, Arqueológico en la Comunidad de San Antonio municipio Pedraza del estado Barinas. La misma estará enmarcada en el paradigma cualitativo y el método a utilizar será la Investigación-Acción Participativa con sus respectivas fases: Diagnóstico, Planificación, Ejecución, Evaluación y Conclusiones. El universo de estudio estará conformado por la comunidad ubicada en el contexto de estudio; los informantes claves los constituirán (1) dueño de finca, un (1) integrante del consejo comunal y un (1) miembro de la comunidad del sector mencionado. Las técnicas de recolección de información que se emplearán serán la entrevista semi estructurada y la observación participante a través de guiones. El análisis de la información se desarrollará a través de la categorización, estas categorías se generarán a medida que se revisa el material, emergiendo del significado de cada sector, evento, hecho dato y la teorización. La validez y fiabilidad se establecerá a través de un exhaustivo proceso de triangulación a fines de establecer acciones concretas para solventar la debilidad detectada en relación a la necesidad de Implementar un plan acción para la preservación del paisaje cultural, arqueológico en la comunidad de San Antonio municipio Pedraza del estado Barinas.

Palabras clave: preservación; paisaje cultural; arqueológico. 


\title{
Preservation of the Archaeological Cultural Landscape in the Community of San Antonio Municipio Pedraza Year 2017
}

(Project execution)

\begin{abstract}
The present study will aim to develop an action plan for the Preservation of the Cultural, Archaeological Landscape in the Community of San Antonio Pedraza municipality of the Barinas state. It will be framed in the qualitative paradigm and the method to be used will be Participatory Action Research with its respective phases: Diagnosis, Planning, Execution, Evaluation and Conclusions. The study universe will be composed of the community located in the study context; The key informants will be (1) farm owner, one (1) member of the community council and one (1) member of the community of the sector mentioned. The information gathering techniques that will be used will be the semi structured interview and the participant observation through scripts. The analysis of information will be developed through categorization, these categories will be generated as the material is reviewed, emerging from the meaning of each sector, event, fact and theorizing. The validity and reliability will be established through an exhaustive triangulation process in order to establish concrete actions to address the weakness detected in relation to the need to implement a plan action for the preservation of cultural, archaeological landscape in the community of San Antonio municipality Pedraza of the state Barinas.
\end{abstract}

Keywords: preservation; cultural; archaeological landscape. 


\section{Introducción}

En los inicios del siglo XXI, la noción del patrimonio cultural no ha cesado de enriquecerse con un enfoque global antropológico y sociológico que lleva a considerarlo como un conjunto de manifestaciones diversas, que se han recibido del pasado, que han llegado a ser testimonios insustituibles representando el desarrollo de una sociedad y, se debe trasmitir a las futuras generaciones. Según Martin (2002) el patrimonio cultural "son elementos y valores culturales, que son compartidos por un grupo humano y los identifica, modificándose en el tiempo" (pág. 42). Para el autor, el hombre como un ser social requiere de una identidad; cada grupo humano posee una identidad cultural, la misma se conforma por elementos culturales y el patrimonio cultural, esta identidad puede ser local, regional o nacional.

En relación a lo anterior, se puede decir que el patrimonio cultural da señas de lo que es la identidad histórica, es uno de los atributos que da personalidad a cada país, se revela a través de las costumbres, tradiciones, yacimientos arqueológicos, geológicos, paleontológicos, considerados importantes en la construcción histórica de la humanidad, sus orígenes y antecedentes. De igual manera, según Blasco (2009) el patrimonio arqueológico, "es un conjunto formado por bienes e inmuebles de carácter histórico, susceptibles de ser estudiados con metodología arqueológica, hayan sido extraídos o no, y tanto si se encuentra en la superficie o en el subsuelo" (pág. 40).

Se infiere de la definición de la autora, que el patrimonio arqueológico, es sin duda, un patrimonio histórico, son testimonios del pasado, que constituyen una herencia colectiva, de allí la importancia de conservarlos, de transmitir de generación en generación su protección, como aporte de un pueblo a la cultura universal. Su estudio no se limita, a la antigüedad en un marco cronológico, sino, implica además, el comportamiento humano desde sus orígenes, sus fuentes son las evidencias antropogénicas, pero también, 
una amplia información contextual de tipo climático, ecológico, geográfico, que actúa como un marco interpretativo de la cultura y ayuda a entender las respuestas adaptativas del hombre ante su ambiente.

Partiendo de lo expresado anteriormente, se considera incluir el patrimonio arqueológico de Venezuela, que su conservación a través de los años ha estado enmarcada, en leyes como la de Protección y Conservación de Antigüedades y Obras Artísticas de la Nación, promulgada en 1945 y la Ley de Protección y Defensa del Patrimonio Cultural en 1993; es en la Constitución de 1999 que se generan nuevas oportunidades para la conservación patrimonial. De allí que la correcta conservación del paisaje arqueológico necesita de acciones planificadas y potenciarla a través de la participación del colectivo.

Ubicándose en el estado Barinas, allí existen restos arqueológicos de gran relevancia tales como: montículos (elevaciones artificiales de tierra en forma de cono truncado), calzadas (terraplenes artificiales que intercomunican los montículos), y los petroglifos con los surcos de mayor grosor y profundidad de Venezuela. Estas representaciones prehispánicas no son conocidas por la población local turistas y visitantes de la zona. Al respecto, Carvajal (2003) expone acerca de ello, que:

En lo prolongado de las sabanas que miré que lucían hermosísimas como arrebataba la atención la dulce música de los pajarillos alegres, sirviéndose de albergue las floridas ramas y verdes pimpollos de unas explayadas como empinadas ceibas jobos, constituidos estos y aquellas en unas eminencias que a manos compusieron las tropas inmensas de indios caquetíos que se retiraron por esto años cuando la venida de los españoles primero tomaron la tierra de Coro (pág. 56).

Cabe destacar que para la conservación del paisaje. Es necesario encontrar orientar y concientizar a la comunidad para el mantenimiento y mejora del mismo, Por tanto, es posible actuar responsablemente y velar de 
forma conjunta por el uso ordenado y racional del paisaje. Tan sólo es necesario que, antes de emprender una actuación que tiene repercusión en la imagen de nuestro entorno, nos informemos y procuremos buscar las soluciones que más se integren en él.

De igual modo, el área arqueológica de Pedraza, estado Barinas, corresponde al punto donde confluyen la cordillera de los Andes y los llanos altos que se extienden al este de la misma. Es una de las regiones de Venezuela cuyos vestigios de la época prehispánica llamaron la atención de números de investigadores. También estos montículos o construcciones de tierra fueron objeto de interés para los cronistas y exploradores que visitaron los llanos durante la etapa de la conquista y la colonización. En tal sentido en Ciudad Bolivia Municipio Pedraza, se encuentran sitios que presentan montículos o calzadas tales como, Los Naranjos, Santa Rosa, Loma de los Indios, las Monjas, Mijaguas. Entre otros.

De tal manera, la región estuvo habitada, desde los primeros siglos de nuestra era, por poblaciones que habían desarrollado formas sociales complejas, las cuales perduraron hasta el siglo XI. Los estudios establecieron que los montículos de mayor tamaño cumplían con funciones de tipo ceremonial, además de representar un símbolo de rango de los caciques y su élite. Los campos elevados permitieron un desarrollo de la agricultura durante la época lluviosa, así como la comunicación entre los diferentes sectores.

Estas construcciones de tierra se encuentran en los espacios de las actuales haciendas agrícolas y ganaderas. Representan un importante valor arqueológico para el estudio de la distribución y relación espacial de los llanos venezolanos y colombianos, por cuanto están presentes en ambos países. Es importante resaltar que, en la comunidad de San Antonio Municipio Pedraza, los restos arqueológicos que dejaron los antepasados no se les han dado el cuidado y protección que estos yacimientos deben de tener, el sitio está deteriorándose poco a poco por la mano del hombre. Es por ello la necesidad 
de resguardarlos para incentivar a los turistas a visitarlos y que las futuras generaciones gocen de su belleza.

En tal sentido es la falta de divulgación sobre la importancia de estos vestigios y el resguardo de los mismos para que no sean afectados por la mano del hombre, concientizar de manera que estos sitios arqueológicos obtengan el valor que representan para la sociedad.

Cabe destacar que los montículos ubicados en la comunidad de San Antonio de Ciudad Bolivia Municipio Pedraza están siendo afectados por la tala, la quema, explotación agrícola, pecuaria y excavaciones realizadas para extraer piezas arqueológicas, trayendo como consecuencia el deterioro de estos montículos, modificando el paisaje cultural y arqueológico, el cual debe ser resguardado y cuidado.

Las posibles causas, se enmarcan en que el paisaje cultural que se encuentra en la comunidad de San Antonio Municipio Pedraza es visible el deterioro, de los montículos en cuanto a la poca vegetación y el tamaño ha disminuido, es importante aplicar estrategias de sensibilización a los miembros de la comunidad para que ayuden a su protección, Esta situación se deriva por la tala y la quema, también el espacio donde se encuentran estos restos arqueológicos no están resguardados de animales, por lo tanto, no se ha dado la importancia que este sitio merece, es allí donde se deben aplicar acciones para la preservación del paisaje natural de esta comunidad, así como para crear e integrar compromisos y tareas en el fortalecimiento de valores culturales.

De allí surge la iniciativa de la presente investigación, la necesidad de crear un plan acción para la preservación del paisaje cultural arqueológico mediante la sensibilización e informar a las comunidades sobre la belleza del paisaje cultural como atractivo turístico de los visitantes, con la finalidad de darlo a conocer y preservarlo para las generaciones futuras. 
De lo anteriormente planteado surgieron una serie de interrogantes:

¿Qué acciones se realizan para la preservación del paisaje cultural, arqueológico en la comunidad de San Antonio municipio Pedraza del estado Barinas?

¿Qué elementos se estructurarían para diseñar un plan acción en la preservación del paisaje cultural, arqueológico en la comunidad de San Antonio municipio Pedraza del estado Barinas?

¿Es posible la ejecución de un plan acción para la preservación del paisaje cultural, arqueológico en la comunidad de San Antonio municipio Pedraza del estado Barinas?

¿Cuáles serán los resultados obtenidos en la ejecución de un plan acción para la preservación del paisaje cultural, arqueológico en la comunidad de San Antonio municipio Pedraza del estado Barinas?

\subsection{Objetivos de la investigación}

\subsubsection{Objetivo general}

Implementar un plan acción para la preservación del paisaje cultural, arqueológico en la comunidad de San Antonio municipio Pedraza del estado Barinas.

\subsubsection{Objetivos específicos:}

Diagnosticar las acciones que se realizan para la preservación del paisaje cultural, arqueológico en la comunidad de San Antonio municipio Pedraza del estado Barinas.

Diseñar un plan acción para la preservación del paisaje cultural, arqueológico en la comunidad de San Antonio municipio Pedraza del estado Barinas. 
Ejecutar un plan acción para la preservación del paisaje cultural, arqueológico en la comunidad de San Antonio municipio Pedraza del estado Barinas.

Evaluar un plan acción para la preservación del paisaje cultural, arqueológico en la comunidad de San Antonio municipio Pedraza del estado Barinas.

\section{Justificación}

La importancia del patrimonio cultural es un tema poco discutido en nuestra sociedad actual, tanto que el común de la gente ignora cuáles elementos constituyen el patrimonio. Consideramos que este problema se debe a la falta de sensibilización en la educación, y a la poca difusión de lo que constituye el patrimonio en las nuevas generaciones de venezolanos. Ese patrimonio debe ser rescatado y preservado, porque constituye la raíz de nuestra identidad y la memoria de nuestro pueblo como nación.

El Patrimonio Cultural es un elemento esencial del pueblo barinés, cuyo proceso de gestión debe estar destinado a su valoración como bien cultural que retroalimente a los distintos grupos sociales, que contribuya a su desarrollo y transformación social, propiciándose el reconocimiento de una sociedad multiétnica y pluricultural, democrática, participativa y protagónica, atendiendo a los derechos culturales reconocidos en los artículos 99 y 100 de la Constitución de la República Bolivariana de Venezuela (1999).

Es de resaltar el gran valor que tiene los restos arqueológicos en el municipio Pedraza, por ello se debe orientar y sensibilizar a la comunidad en general para la conservación y resguardo de los mismos a través de talleres en las instituciones ya que algunos de estos vestigios están cerca de instituciones educativas.

Desde el punto de vista social, se permitirá informar a la comunidad en general como se debe cuidar y resguardar los restos arqueológicos que fueron 
dejados por los antepasados, permitiendo a las generaciones futuras y visitantes observar y valorar el paisaje cultural de la comunidad.

En lo concerniente a la justificación metodológica, el trabajo se desarrollará a través de la Investigación cualitativa, proponiéndose desde la investigación acción participante, Se aspira que la misma sirva de contribución para crear y aplicar acciones para la preservación arqueológica del paisaje natural.

\section{Antecedentes}

En los antecedentes se recogen los puntos que se deben extraer de cada investigación consultada, objetivos, propósito, teoría, metodología para el desarrollo del trabajo, resultados y conclusiones. Luego de realizar la revisión bibliográfica, se encontraron algunos trabajos, entre los cuales se pueden mencionar:

De igual manera, Valenzuela y Viloria (2011), desarrollaron un estudio investigativo cuyo objetivo persiguió como fin primordial, proponer estrategias metodológicas para la enseñanza del patrimonio cultural local en el área de educación para el trabajo, caso: Unidad Educativa "Juan Bautista Dalla Costa" del Estado Trujillo durante el año escolar 2010-2011. Epistémicamente se ubica en el paradigma humanista y metodológicamente es de tipo cualitativa, enmarcada en el campo de la etnografía escolar, bajo un diseño de enfoque progresivo. La muestra es de tipo intencional y está constituida por nueve informantes claves conformados por directivos, docentes, alumnos y comunidad. También se recolectaron datos de tipo cuantitativo para dar un soporte mayor a la investigación. La información fue recopilada mediante notas de campo, entrevistas semi-estructuradas y la observación participante; la validación fue mediante el método de triangulación. El análisis de resultados determinó que existe conocimiento muy superficial sobre el patrimonio cultural en docentes y alumnos y que no existe una metodología efectiva para la 
enseñanza de valores culturales en el área por lo que se diseñó un plan para solventar las deficiencias detectadas e integrar estrategias innovadoras.

La investigación de Valenzuela y Viloria, se relaciona con la presente tesis porque ambas investigaciones se fundamentan en datos cualitativos correspondientes a promover mediante diversas actividades y estrategias el conocimiento y conservación del patrimonio cultural, en donde el proceso de enseñanza-aprendizaje juega un papel importante que se refleja a través de la toma de conciencia de la comunidad frente a la preservación del entorno cultural donde vive.

\section{Sustento teórico}

\subsection{Preservación arqueológica}

La acción de preservar consiste en cuidar, amparar o defender algo con anticipación, con el objetivo de evitar un eventual perjuicio o deterioro. La preservación, por lo tanto, puede desarrollarse en diversos ámbitos y de distintas formas para conservar las cualidades o la integridad de las cosas.

Por otro lado, la arqueología, es el estudio o rescate de los objetos, del significado de las cosas, Navarrete (2004) destacó que: "la arqueología es la rama que le da significado a las cosas, ya que cada objeto cuenta una historia importante del patrimonio venezolano" (pág. 224). Asimismo, los objetos cuentan la historia que necesitamos nosotros para comprender la historia de Venezuela y del mundo.

Es necesario resaltar la importancia de preservar nuestro legado arqueológico, ya que a través de él se puede contar la diversidad de tradiciones culturales presentes en territorio venezolano. Por estas razones se debe despertar en la sociedad, el sentido de pertenencia del patrimonio arqueológico. 


\subsection{Acciones conservacionistas}

El término acción se refiere al acto de hacer algo, cuyo propósito es influir a otros para que estos participen en la resolución de una situación problemática ambiental. Se puede hablar de acciones directas e indirectas, pero ambas están muy relacionadas debido a que una acción ambientalista indirecta, como por ejemplo una demostración pública o una campaña de información puede ayudar al desarrollo de tareas directas que mejora el entorno.

En este sentido, las acciones pueden también ser individuales $\mathrm{y} / \mathrm{o}$ colectivas, pero en ambas hay un compromiso personal para el desarrollo de una tarea que se hace a nivel individual en la cual la participación es singular o a nivel público o colectivo donde la acción se plantea en un escenario social. También es posible clasificar las acciones con relación al tratamiento del problema ambiental así es posible hablar de acciones preventivas de igual forma de acciones correctivas.

\subsection{Protección del Medio Ambiente}

La importancia de la protección del medio ambiente reside en que sin ella, muchos de los bienes materiales y naturales con los que cuenta la sociedad actualmente, no existirían en el futuro. Por tanto, como seres racionales, se debe incluir esta preservación del patrimonio como una de las prioridades morales, siendo cuidadosos con el patrimonio histórico, cultural y natural que dejaron anteriores generaciones. Es entonces como la preservación puede proteger los paisajes y animales que rodea al ser humano, quizá este sea uno de los trabajos más importantes de este concepto, pues el hombre está acostumbrado a vivir en las grandes ciudades, dejando muchas veces de lado el mundo natural.

Así, se crean gran variedad de programas a raíz de esta intención, iniciativas que sirven para poder cuidar de determinadas especies y por 
ejemplo facilitar su reproducción cuando se habla de especies animales, pero de recursos naturales. De esta manera, Barazarte (2012) expresa: "También se intentan cuidar algunos paisajes que están siendo dañados por el cambio climático o incluso por la propia mano del hombre, como los paisajes con problemas de deforestación." (pág. 12). En la preservación natural, hay un epígrafe muy importante que se refiere a la preservación del medio ambiente.

\section{Naturaleza de la Investigación}

El estudio está sustentado en el enfoque cualitativo, dado que trata de comprender a las personas dentro del marco de referencia de ellas mismas, de manera que es esencial experimentar la realidad tal como otros la experimentan. Según Martínez (2010) afirma que "la investigación cualitativa trata de identificar la naturaleza profunda de las realidades, su estructura dinámica" (pág. 18). Lo que se desea es el análisis de forma detallada de un asunto o actividad particular, es decir; lo que se busca es solucionar de forma detallada la situación o problemática estudiada.

\section{Método o tipo de Investigación}

La investigación, se enfoca en el método Investigación Acción Participante, como lo argumentan Hurtado y Toro, (2005) "esta modalidad se relaciona con los problemas prácticos y cotidianos experimentados por los profesores, en vez de hacerlo con los problemas teóricos definidos por los investigadores puros en el entorno de una disciplina del saber" (pág. 56).

De acuerdo a lo antes citado, por los autores la investigación acción, nos permite participar activamente en la misma como sujeto de estudio, para conocer la realidad de e implementar estrategias conservacionistas para la preservación de los montículos como patrimonio arqueológico, En tal sentido Martínez (2006) establece que: 
La investigación- acción realiza simultáneamente la expansión del conocimiento científico y la solución de un problema, mientras aumenta, igualmente, la competencia de sus respectivos participantes (sujetos coinvestigadores) al ser llevada a cabo en colaboración, en una situación concreta y usando la realimentación de la información en un proceso clínico (pág. 239).

Por consiguiente la investigación Acción, propone desarrollar una labor que contribuya a conocer a fondo la situación, a transformar una realidad, pero fundamentalmente a conocer las experiencias de las personas involucradas, haciendo de la investigación un proceso de reflexión crítica, donde se concibe al sujeto como ser activo y transformador de su entorno, debido que la información que se obtenga de la realidad, permita Implementar acciones conservacionistas para la preservación del paisaje cultural arqueológico.

\section{Fases de la Investigación Acción}

Para la estructura de la investigación acción participativa, se toma como referencia las fases propuestas por Martí (2003):

8.1. Primera Fase. Diagnóstico: en esta fase se aplicarán las técnicas para recolectar la información, pertinente sobre la problemática que se hace presente en el ámbito comunitario, específicamente para describir los factores internos, externos y conocer los agentes que condicionan la participación de los integrantes de la comunidad, en la preservación del paisaje cultural arqueológico.

8.1.1. Segunda Fase. Planificación: en esta fase se planificarán las estrategias a partir del diagnóstico, para involucrar a la comunidad de San Antonio, en las actividades, tendientes a preservar el paisaje cultural como patrimonio arqueológico.

8.1.2. Tercera Fase. Ejecución: fase en la cual, se ejecutarán las acciones, éstas son desarrolladas por los actores interesados en el proceso 
de preservación arqueológico; con las estrategias planificadas, donde se permitirán las orientaciones para participar en las actividades comunitarias, siendo necesarias la negociación y el compromiso, para fortalecer el valorar el patrimonio arqueológico del Municipio Pedraza.

8.1.3. Cuarta Fase. Evaluación: la evaluación marca el impacto de las estrategias ejecutadas, en este orden de ideas, esta evolución se fundamentará en un enfoque cualitativo, el cual se acciona del contexto donde se producirá la acción, un espacio para la reflexión, compresión y valoración de los avances, intereses aspiraciones, consideraciones e interpretaciones de la preservación arqueológica como patrimonio cultural y científico.

8.1.4. Quinta Fase. Conclusiones: consiste en la reflexión sobre todo el trabajo a realizarse, con el fin de obtener conclusiones que sirvan para el futuro. En las fases antes mencionadas se permitirá la participación activa de los actores involucrados, promoviendo estrategias para incentivar a la preservación del paisaje cultural arqueológico.

\section{Técnica de Recolección de la Información}

Es importante definir en toda investigación lo relacionado con los métodos, instrumentos y técnicas de recolección de información que se utilizarán durante el proceso de investigación en función con el planteamiento del problema, la interrogante y los objetivos planteados. La metodología cualitativa entiende el método y todo el arsenal de medios instrumentales como algo flexible, que se utilizará mientras resulte efectivo, pero que puede cambiar de acuerdo a lo imprevisto, según la marcha y circunstancia. (Martínez, 2006).

Para efecto, la obtención de la información, se recurrirá a la técnica de la entrevista según Palella (2006: 35), "es una técnica que permite obtener datos mediante un dialogo que se realizan entre dos personas cara a cara: El investigador y el entrevistado; la intención es obtener información que posee 
a este último". Mediante la aplicación de esta técnica se podrá identificar las dificultades que originan la problemática que se desea cambiar o mejorar, a través de una guía de entrevista orientada hacia el análisis, la reflexión, y posteriormente interpretar los hechos.

\section{Técnicas y Análisis de la Información}

Es uno de los momentos más importantes del proceso, e implica que una vez recogida la información se realizará una descripción cualitativa de las acciones manifestadas, según los aspectos indicados e interpretados; de igual manera se efectuará un análisis interpretativo de la información para tratar de explicar y crear generalizaciones, refinar conocimientos, identificar problemas, clarificar y comprender la complejidad del problema planteado.

Las técnicas y análisis de la información, permite procesar la información obtenida en las guías temáticas de la entrevista en profundidad, pero la misma debe ser organizada y esquematizada, trascribiendo todos los contenidos, "entendiéndose como los relatos escritos, extraídos de las grabaciones y realidades de las situaciones concretas”. Martínez (2006). Por lo tanto, se debe organizar la información de manera escrita citando las fuentes de las mismas y las diversificaciones que se desprenden.

\subsection{Categorización}

En las investigaciones cualitativas, la información obtenida de la aplicación de las técnicas descritas anteriormente, requieren ser analizadas mediante la categorización e interpretación de los contenidos. Por esta razón la información obtenida se categorizará, de acuerdo a Martínez (2006), como:

Categorizar o clasificar las partes en relación con el todo, de describir categorías 0 clases significativas, de ir constantemente diseñando y rediseñando, integrando y reintegrando el todo y las partes, a medida que se revisa el 
material y va emergiendo el significado de cada sector, evento, hecho dato (pág. 266).

En cuanto a la categorización, es pertinente analizar, interpretar y describir la información suministrada por los informantes claves, para obtener los resultados de manera clara y precisa, verificando que dichos resultados tengan relación con los objetivos planteados en la investigación.

\subsubsection{Triangulación}

Es una técnica para analizar los datos cualitativos, lo cual permite analizar una situación de diversos ángulos. Es un control cruzado empleando diferentes fuentes, instrumentos o técnicas de recogida de datos. La triangulación como una de las técnicas más empleadas para el procesamiento de los datos en las investigaciones cualitativas.

En relación a la triangulación como técnica de enfoque cualitativo, Bermejo, (2005) manifiesta: "Que es la recogida de datos desde distintos ángulos para compararlos y contrastarlos entre sí. Control cruzado entre diferentes fuentes de datos: Personas, instrumentos, documentos, teorías, métodos y la combinación de ellos". (pág. 27).

En este mismo sentido, en esta técnica se puede obtener una interpretación más completa, comprensiva y objetiva del hecho o acontecimiento estudiado, Dando el cruce de los datos suministrados por los informantes claves, las teorías de autores que sustentan la información y por ende las apreciaciones del investigador.

\section{Validez de la Investigación}

El estudio con su carácter de investigación cualitativa se tomará como método de validez, la triangulación, que según Elliott (2000), consiste en "recoger observaciones y apreciaciones de una situación o algún aspecto de 
ella desde una variedad de ángulos o perspectivas después de compararlas y contrastarlas" (pág. 103). Todo esto con la finalidad de determinar ciertas intersecciones o coincidencias a partir de diferentes apreciaciones y fuentes informativas o varios puntos de vista del mismo fenómeno.

Al respecto Martínez (2006), sostiene que:

La validez es la fuerza mayor de la investigación cualitativa. En efecto, el modo de recoger los datos, de captar cada evento desde sus diferentes puntos de vista, de vivir la realidad estudiada y de analizarla e interpretarla inmersos en su propia dinámica, ayuda a superar la subjetividad y da a estas investigaciones un rigor y una seguridad en sus conclusiones que muy pocos métodos pueden ofrecer. (pág. 255).

De acuerdo a lo antes expuesto, en la investigación cualitativa, la validez es la de mayor peso en la investigación, porque permite recoger las informaciones y captar cada escenario desde el punto de vista de estudiar, analizar e interpretar la realidad proporcionando la fiabilidad de las conclusiones.

\section{Fiabilidad de la Investigación}

La fiabilidad implica que un estudio se puede repetir con el mismo método sin alterar los resultados, es decir, es una medida de la replicabilidad de los resultados de la investigación.

Para Martínez (2006) "la fiabilidad está orientada hacia el nivel de concordancia interpretativa entre diferentes observadores, evaluadores 0 jueces del mismo fenómeno, es decir, la fiabilidad será sobre todo interna, ínterjueces" (pág. 256). De acuerdo a lo antes descrito, la fiabilidad es cuando los informantes clave, poseen muchas coincidencias en las respuestas, dando un alto índice de confianza. 


\section{Conclusiones}

No se han podido obtener los resultados pertinentes, por ser esta una investigación en curso, pero se puede discernir que a través del desarrollo del plan acción para la preservación del paisaje cultural, arqueológico en la comunidad de San Antonio municipio Pedraza del estado Barinas se puede lograr preservar muchos de los bienes culturales, arqueológicos y naturales con los que cuenta el municipio Pedraza actualmente, para que puedan existir en el futuro. Por tanto, se deben incluir estrategias para lograr esta preservación del patrimonio como una de las prioridades morales, siendo cuidadosos con el patrimonio histórico, cultural y natural que dejaron anteriores generaciones. De allí, es importante concienciar a la sociedad de que, si no se cuida el planeta, seguramente seremos los humanos los que no puedan vivir aquí. Así pues, esta preservación medioambiental no es más que una preservación de nuestra propia especie.

Es por ello la necesidad de ejecutar esta investigación en la cual se puede consumar lo siguiente: Investigar y promover acciones en torno a la preservación del paisaje cultural, arqueológico en la comunidad de San Antonio municipio Pedraza del estado Barinas, desde un contexto ambiental. De igual manera, promover el conocimiento de la problemática de la conservación del paisaje cultural, arqueológico en sus múltiples facetas.

\section{Referencias}

Blasco, M. (2009). Patrimonio Histórico. Edición de Universidad de Cantabria. Tercera edición. España.

Carvajal, A. (2003). Paisaje Patrimonial y Riesgo Ambiental. Universidad de San Sebastián. Chile.

Constitución Nacional de la República Bolivariana de Venezuela (1999).

Gaceta Oficial de la República de Venezuela (Extraordinaria), $\mathbf{N}^{\circ}$ 36.860, de fecha 30 de diciembre. Caracas, Venezuela. 
Elliott, J. (2000). El cambio educativo desde la investigación acción. Madrid.

Hurtado, I. Toro, J. (2005). Paradigma y métodos de investigación en tiempos de cambio. Los libros de El Nacional. Colección Minerva.

Martínez M. (2006). Ciencia y arte en la metodología cualitativa. México: Trillas.

Navarrete, A. (2004). Cultura y Patrimonio. Biblioteca básica temática. Consejo Nacional d la cultura. Caracas, Venezuela.

Valenzuela, T., y Viloria, N. (2011). Estrategias metodológicas para la enseñanza del patrimonio cultural local en el área de educación para el trabajo. Caso: unidad educativa "Juan Bautista Dalla Costa" del municipio Boconó del Estado Trujillo. Año Escolar 2010 -2011. Trabajo de Investigación y Postgrado como requisito para optar al título de Magister en Educación, Ambiente y Desarrollo. [Tesis Doctoral publicada en Revista Científica].

Goetz J., y LeCompte M. (2003). Etnografía y Diseño Cualitativo en Investigación Educativa. Morata. Madrid. 


\section{Isabel Galeano Torres}

e-mail:22marije@gmail.com

Técnico superior en informática. Instituto universitario

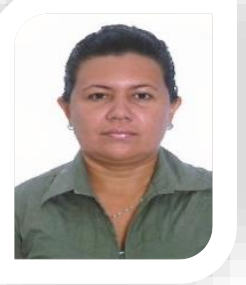
de tecnología Coronel Agustín Codazzi año 2002. Lcda. En Educación Integral. Universidad Experimental de los Llanos Occidentales Ezequiel Zamora (UNELLEZ). Año 2008. Curso Operador de computadora año 1998. Participación en el I encuentro Regional Sobre Calendario Productivo año 2003. Jornada de Orientación y concientización del área deficiencia visuales año 2004. Participación en el $21^{\circ}$ Encuentro Nacional y 5 to Internacional de Educadores y Educadoras año 2005. Asistencia "Como formar niños y niñas lectoras y Escritores Autónomos" en el $21^{\circ}$ Encuentro Nacional y 5to Internacional de Educadores y Educadoras año 2005. I jornada de planificación y evaluación del proceso educativo. Año 2005. Taller "Conociendo la Ley Resorte" año 2007. Curso de primeros auxilios año 2009. Curso en "Actualización o Perfeccionamiento Profesional de IV Nivel con Evaluación". Docente de Aula 2003 - 2012 N.E.R 001, Coordinadora de Cultura 2012 - 2016 N.E.R 001 Docente de Aula 2016 hasta la actualidad en Ciudad Bolivia Municipio Pedraza Estado Barinas. 